\title{
An Amyloidogenic Determinant in N-terminal Pro-Brain Natriuretic Peptide (NT-proBMP): Implications for Cardiac Amyloidoses
}

Vassiliki A. Iconomidou, ${ }^{1}$ Danae Pheida, ${ }^{1}$ Eftihia S. Hamodraka, ${ }^{1}$ Claude Antony, ${ }^{2}$ Andreas Hoenger, ${ }^{3}$ Stavros J. Hamodrakas ${ }^{1}$

${ }^{1}$ Department of Cell Biology and Biophysics, Faculty of Biology, University of Athens, Panepistimiopolis, Athens 157 01, Greece

${ }^{2}$ EMBL Heidelberg, Meyerhofstraße 1, 69117 Heidelberg, Germany

${ }^{3}$ Department of Molecular Cellular and Developmental Biology, University of Colorado at Boulder, Boulder, CO 80309-0347

Received 12 March 2011; revised 23 May 2011; accepted 6 July 2011

Published online 25 July 2011 in Wiley Online Library (wileyonlinelibrary.com). DOI 10.1002/bip.21698

\begin{abstract}
:
Deposition of amyloid in the atria (isolated atrial/cardiac amyloid) is fairly common in the aging heart. It consists of amyloid fibrils, formed both by atrial natriuretic peptide (ANP) and brain natriuretic peptide (BNP) and the precursor molecule of ANP, proANP. This study examines whether amyloidogenic determinants (short peptides/amyloid forming favoring regions) exist in the sequence of NT-proBNP, the N-terminal part of proBNP, and if these determinants form amyloid-like fibrils in vitro. We have predicted a possible amyloidogenic determinant in the sequence of the NT-proBNP, and we conclusively show, after its synthesis, that it forms amyloid-like fibrils in vitro, utilizing transmission electron microscopy, X-ray diffraction, attenuated total reflectance Fourier-transform infrared spectroscopy, and polarizing microscopy. Thus, for the first time, in this study, a possible biological role is attributed to a certain, specific part of this important cardiac prohormonel natriuretic peptide, which acts as an important biomarker indicative of heart failure. Its possible direct involvement in isolated cardiac amyloidosis, atrial fibrillation, and other types of cardiac amyloidoses is
\end{abstract}

Correspondence to: Stavros J. Hamodrakas, Department of Cell Biology and Biophysics, Faculty of Biology, University of Athens, Panepistimiopolis, Athens 157 01, Greece; e-mail: shamodr@biol.uoa.gr (C) 2011 Wiley Periodicals, Inc. indicated and discussed. Since these cardiac hormones and their prohormones play key roles in cardiovascular homeostasis through natriuresis, diuresis, vasorelaxation, and inhibition of renin and aldosterone secretion (pathophysiology of hypertension and cardiovascular regulation), we also try to suggest these specific, short peptides as possible future structural targets of efforts toward inhibiting formation of natriuretic peptide(s) amyloid. (C) 2011 Wiley Periodicals, Inc. Biopolymers (Pept Sci) 98: 67-75, 2012.

Keywords: natriuretic peptides; $N$-terminal pro-brain natriuretic peptide (NT-proBNP); amyloid-like fibrils; cardiac amyloidoses; isolated atrial amyloidosis

This article was originally published online as an accepted preprint. The "Published Online" date corresponds to the preprint version. You can request a copy of the preprint by emailing the Biopolymers editorial office at biopolymers@wiley. com

\section{INTRODUCTION}

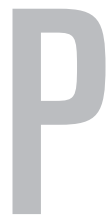

roteins or peptides convert under certain conditions from their soluble forms into ordered fibrillar aggregates called amyloid fibrils. Protein aggregation and occasionally ensued amyloid fibril formation are believed to be the cause of an intriguing group of neurodegenerative diseases, including Alzheimer's disease, Parkinson's and Huntington's disease, and, also, type II diabetes, prion diseases, and many others, that are referred to as 
amyloidoses. ${ }^{1,2}$ Tissue deposition of insoluble amyloid fibrils can result to organ dysfunction and death. However, occasionally, living organisms take advantage of the inherent ability of proteins and peptides to form such structures under certain conditions and generate novel and diverse biological functions. $^{3,4}$ Attention to these functional amyloids was given after our finding that natural protective amyloids, indeed, exist. ${ }^{5,6}$

Cardiac amyloidosis is a relatively common finding in elderly patients, 70-80 years or more of age. There are two types of cardiac amyloidoses. The first type is more frequent and involves the atria (isolated cardiac/atrial amyloidosis), whereas the second type involves both the atria and the ventricles (senile cardiac amyloidosis). In the case of isolated atrial amyloidosis (IAA), studies have shown that natriuretic peptides such as atrial natriuretic peptide (ANP), brain natriuretic peptide (BNP), and the precursor molecule of ANP, proANP, are major subunits of the amyloid fibrils. ${ }^{7,8}$ The main clinical consequences of amyloid deposition in the heart are cardiac failure and rhythm disturbances. ${ }^{9}$ In general, hearts with cardiac amyloidosis preserve systolic function until the terminal stage, but diastolic function is damaged, mainly as a result of the mechanical disturbance of myocyte movement by the diffuse deposition of amyloid. ${ }^{10}$

Immunohistochemical studies have indicated the presence of ANP in isolated atrial amyloid, which is deposited on the surface of atrial myocytes. ${ }^{11}$ It has been proven that ANP is the major low molecular weight protein in atrial amyloid. Such amyloid deposits are present in isolated cardiac/atrial amyloidosis. ${ }^{7}$ ANP occurs specifically in the tissue of origin, the atrium of the heart. ${ }^{7}$ ANP is an unstable molecule that is degraded and rapidly eliminated from the circulation, ${ }^{12}$ but when it occurs in amyloid state, ANP appears to be a very stable molecule.

ANP was the first cardiac hormone to be identified. ${ }^{13-15}$ Later, another hormone, BNP, which is synthesized and secreted mainly from the heart, was identified. ${ }^{16}$ The BNP is a peptide hormone, which is synthesized in atrial and to a greater extent in ventricular cardiomyocytes. ${ }^{17,18}$ The name BNP was given because of the fact that it was originally isolated from porcine brain tissue. ${ }^{16}$ ANP and BNP are in the same natriuretic peptide family and have a homologous structure and activity. They control the body fluid and electrolyte balance, causing diuresis, vasodilatation, and decreased rennin and aldosterone secretion. ${ }^{19}$

Immunoreactivity studies have proven the presence of both ANP and BNP immunoreactivity in atrial amyloid fibrils. ${ }^{8}$ In contrast, neither ANP nor BNP has been detected until now in the ventricular amyloid fibrils in cardiac amyloidosis. ${ }^{10}$ These studies also showed that ANP and BNP and the precursor peptide proANP are constituents of amyloid fibrils of IAA. $^{8}$

BNP is synthesized as a 134-aminoacid polypeptide, which then becomes a 108-aminoacid polypeptide prohormone BNP (proBNP) after cleavage of the signal peptide. The mature form of BNP consisting of 32 amino acid residues is included in the carboxyl terminus of proBNP. ${ }^{20,21}$ The proteolytic enzymes furin and corin are able to catalyze this step, and the prohormone cleavage is thought to occur during the process of peptide secretion. The process from proBNP to mature BNP, the secretion of BNP and also the clearance of BNP from plasma, is not fully understood yet. $^{22,23}$ NT-proBNP (amino terminal proBNP) is a 76amino acid residue polypeptide, which remains after the cleavage of mature BNP (32 amino acid residues) from the prohormone, proBNP (see Figure 1).

Both BNP and NT-proBNP are extensively used as biomarkers for diagnosis of ventricular dysfunction in heart failure patients. ${ }^{24-28}$ BNP synthesis and secretion is activated by cardiac myocyte stretch. In heart failure, increased wall stretch (due to both volume and pressure overload), neurohormonal activation, and hypoxia stimulate BNP secretion. Decline in the ventricular function elevates concentration of BNP, and therefore, measuring the concentration of BNP can be a useful marker for the diagnosis. BNP is cleared rapidly from circulation, ${ }^{29}$ resulting in a higher plasma concentration of NT-proBNP. ${ }^{30}$ In addition, NT-proBNP has a longer half-life $\left(120 \mathrm{~min}^{31,32}\right)$ than BNP $\left(20 \mathrm{~min}^{32,33}\right)$. The longer half-life for the NT-proBNP peptide may be related to its larger size and greater reliance on renal function for clearance. ${ }^{34}$ NT-proBNP is therefore used as a more sensitive biomarker for detection and evaluation of heart failure.

In the case of cardiac amyloidosis, the expression of both ANP and BNP is augmented in the ventricular myocytes and particularly in regions adjacent to amyloid deposits. Hemodynamic stress accompanied by ventricular diastolic dysfunction and regional mechanical stress restricting myocardial movement by amyloid deposits may be important triggers for the overexpression of these peptides in patients with cardiac amyloidosis. The elevation of the secretion of BNP is more pronounced than that of ANP. ${ }^{10}$

Excellent recent reviews describe the biology, biochemistry, pathophysiology, peptide release, bioactivity, clinical uses, and mechanisms of clearance of natriuretic peptides. ${ }^{18,32,35-42}$ In general, our attention was drawn to NTproBNP because of the importance of this molecule as a biomarker for cardiac amyloidoses and heart failure. In AL amyloidosis (primary systemic amyloidosis) in which amyloid fibrils are composed mostly by the N-terminus of a monoclonal immunoglobulin light chain, NT-proBNP is considered 
Signal peptide

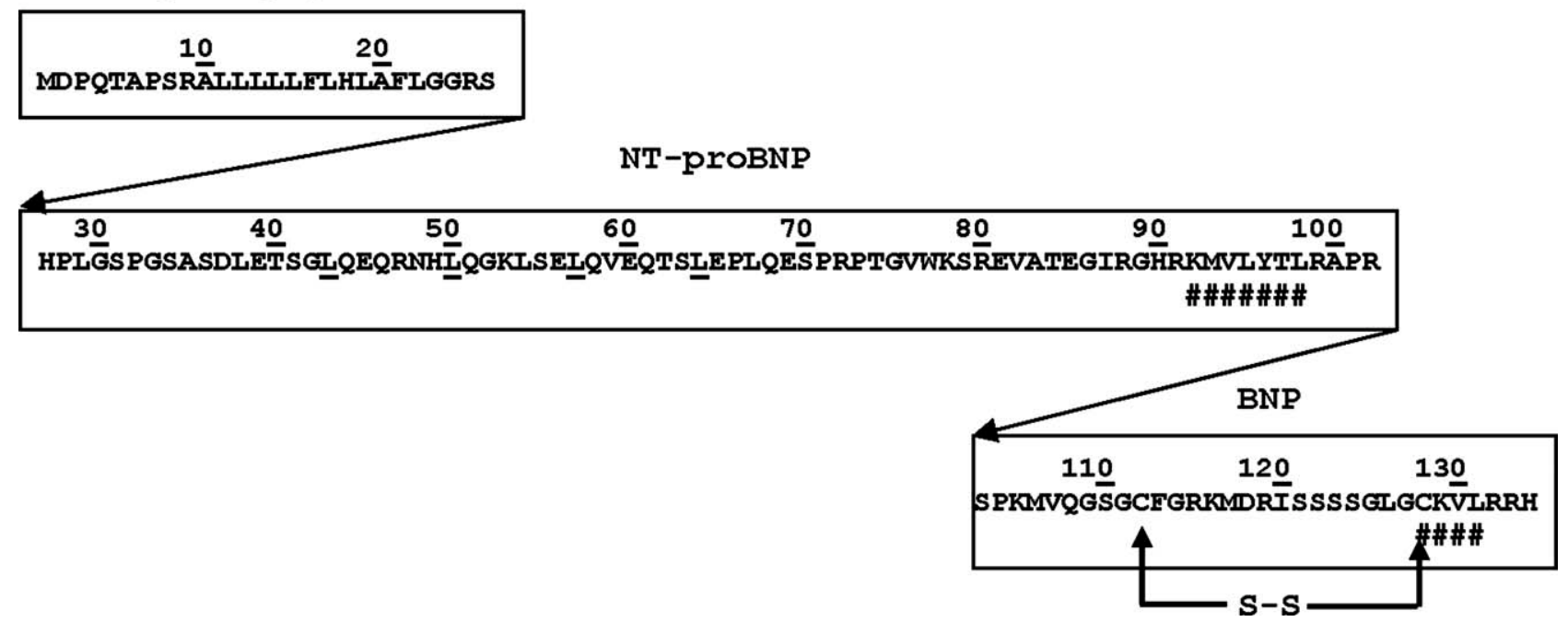

FIGURE 1 A schematic representation of the aminoacid sequence of brain natriuretic peptide (BNP) is shown. BNP is synthesized in atrial and to a greater extent in ventricular cardiomyocytes $^{18}$ as a 134 -aminoacid polypeptide, which then becomes a 108 -aminoacid polypeptide prohormone BNP (proBNP) after cleavage of the 26-residue signal peptide (Signal peptide, residues 1-26). The mature form of BNP, which consists of 32 amino acid residues (residues 103-134), is included in the carboxyl terminus of proBNP (BNP). The proteolytic enzymes furin and corin are able to catalyze this step. NT-proBNP (amino terminal proBNP) is a 76-amino acid residue polypeptide (residues 27-102), which remains after the cleavage of mature BNP (32 amino acid residues) from the prohormone, proBNP. The biologically active 32-residue BNP, the intact 108 amino acid proBNP, and the 76-residue NT-proBNP all circulate in the plasma and can be measured by immunoassay. There is a disulfide bridge between Cys113 and Cys128 in mature BNP, essential for the biological action of mature BNP, which is shown by arrows below the sequence. Predicted amyloidogenic determinants by our algorithm AMYLPRED ${ }^{47}$ for NT-proBNP and BNP are marked by "\#” below the sequence. There are two such determinants: one is the heptapeptide KMVLYTL at the C-terminal of NT-proBNP (residues 92-98), and the other is the tetrapeptide CKVL at the C-terminal of BNP (residues 128-131). The underlined four leucines $(L)$ in the Nterminal portion of NT-proBNP (residues 43-64) indicate the part of NT-proBNP considered to be a candidate for the formation of leucine zipper-like coiled-coil motifs, that is $\alpha$-helices wound around each other into a superhelix, thus promoting NT-proBNP oligomerization. ${ }^{75}$

to be the most powerful prognostic factor. ${ }^{43}$ Furthermore, the exact biological role of NT-proBNP remains unclear. ${ }^{35-37}$

Evidence indicates that short sequence stretches may be responsible for amyloid formation, ${ }^{44-46}$ and several methods have been published recently, which attempt to predict these amyloidogenic regions, hereinafter called amyloidogenic determinants, based on various properties of proteins. ${ }^{47} \mathrm{We}$ have developed a consensus prediction algorithm called AMYLPRED ${ }^{47}$ which combines several amyloidogenic determinant prediction algorithms, freely accessible to academic users at http://biophysics.biol.uoa.gr/AMYLPRED, and we have shown that this tool successfully predicts, nearly all, experimentally verified amyloidogenic determinants in the sequences of proteins causing amyloidoses. ${ }^{47}$ Furthermore, AMYLPRED predicts on the sequences of amyloidogenic proteins several short potential amyloidogenic stretches, not yet experimentally verified as such. ${ }^{47}$
Our aims are to (a) to test the validity of our consensus prediction algorithm and see how many of the predicted but not yet experimentally verified amino acid stretches have indeed amyloidogenic properties and (b) to study the origins of IAA, which apparently are related to natriuretic peptides ANP and BNP and their precursor molecules proANP and proBNP. ${ }^{7,8,48}$ Therefore, in this study, the algorithm AMYLPRED was applied to the proBNP sequence (see Figure 1); one predicted amyloidogenic determinant heptapeptide was synthesized (see the "Materials and Methods" section), and here, we present experimental results verifying that this predicted amyloidogenic determinant heptapeptide of NT-proBNP is indeed an amyloidogenic determinant. We discuss the implications of our findings to IAA and may be to cardiac amyloidoses in general, and we try to explain its involvement in molecular terms and to propose possible remedies. 


\section{METHODS}

\section{Prediction of Amyloidogenic Determinants in proBNP}

Our algorithm AMYLPRED, ${ }^{47}$ which is a consensus prediction tool of amyloidogenic determinants (short stretches of amino acid sequences in the primary structure of proteins responsible for amyloid formation), combining five, independent amyloidogenic determinant prediction algorithms, freely accessible to academic users at http://biophysics.biol.uoa.gr/AMYLPRED, was applied on the amino acid sequence of proBNP (NT-proBNP and BNP, Figure 1), obtained from UNIPROT (accession number P16860). Two possible amyloidogenic determinants were predicted (see Figure 1): The first, a heptapeptide, KMVLYTL, (nominal positions 66-72), in the C-terminal portion of NT-proBNP (76 residues in length, Figure 1) and the second, a tetrapeptide CKVL (nominal positions 26-29), in the C-terminal segment of mature BNP (32 residues in length, Figure 1).

\section{Peptide Synthesis}

The NT-proBNP heptapeptide-analogue KMVLYTL was synthesized by GeneCust Europe, Luxembourg (purity $>98 \%$, free N- and C-terminals). We did not synthesize the tetrapeptide CKVL, apparently an amyloidogenic determinant in the sequence of mature BNP, and we did not test yet its amyloidogenic properties, because the presence of a highly reactive Cys residue in its sequence (residue 26 in mature BNP, Figure 1) would cause difficulties in the experiments by the formation of intermolecular disulfide bonds. It is well known that Cys26 in the sequence of mature BNP forms an intramolecular disulfide bridge with Cys $10,{ }^{35,49}$ which is important for the structure and biological function of BNP.

\section{Formation of Amyloid-Like Fibrils}

The synthesized NT-proBNP heptapeptide-analogue, KMVLYTL, was dissolved in doubly distilled water ( $\mathrm{pH}$ 5.5), at a concentration of $15 \mathrm{mg} \mathrm{ml}^{-1}$. Mature amyloid-like fibrils were formed after 1-2 weeks incubation, forming fibril-containing gels. The fibrils were judged to be mature, observing preparations both for shorter and longer periods than 1-2 weeks. Oriented fibers, suitable for X-ray diffraction, were obtained from solutions of the peptide, containing mature amyloid-like fibrils as described below.

\section{X-ray Diffraction}

The NT-proBNP heptapeptide-analogue, KMVLYTL, was dissolved in doubly distilled water ( $\mathrm{pH}$ 5.5) at a concentration of $15 \mathrm{mg} \mathrm{ml}^{-1}$ to produce mature amyloid-like fibrils after 1-2 weeks incubation forming a fibril-containing gel. A droplet $(\sim 10 \mu \mathrm{l})$ of fibril suspension was placed between two siliconized glass rods, spaced $\sim 2$-mm apart and mounted horizontally on a glass substrate, as collinearly as possible. The droplet was allowed to dry slowly at ambient temperature and humidity for $1 \mathrm{~h}$ to form an oriented fiber suitable for X-ray diffraction. $\mathrm{X}$-ray patterns were obtained immediately from these fibers, since it was found that fiber orientation was lost after $\sim 2 \mathrm{~h}$ under these conditions. X-ray diffraction patterns were recorded on a Mar Research 345-mm image plate, utilizing $\mathrm{CuK}_{\alpha}$ radiation $(\lambda=1.5418 \AA$ ), obtained from a Rigaku MicroMax-007 HF, microfocus rotating an- ode generator (with Osmic Rigaku VariMax ${ }^{(i m}$ HF optics), operating at $40 \mathrm{kV}, 20 \mathrm{~mA}$. The specimen-to-film distance was set at $150 \mathrm{~mm}$, and the exposure time was $30 \mathrm{~min}$. No additional low-angle reflections were observed at longer specimen-to-film distances of up to $300 \mathrm{~mm}$. The X-ray patterns, initially viewed using the program MarView (MAR Research, Hamburg, Germany), were displayed and measured with the aid of the program IPDISP of the CCP4 package. ${ }^{50}$

\section{Negative Staining}

For negative staining, the NT-proBNP heptapeptide-analogue, KMVLYTL, fibril suspensions after incubation for 1-2 weeks were applied to glow-discharged 400-mesh carbon-coated copper grids for $60 \mathrm{~s}$. The grids were (occasionally) flash-washed with $\sim 150 \mu \mathrm{l}$ of distilled water and stained with a drop of $1 \%(\mathrm{w} / \mathrm{v})$ aqueous uranyl acetate for $45 \mathrm{~s}$. Excess stain was removed by blotting with a filter paper, and the grids were air-dried. Pictures were acquired in a Philips CM120 BioTWIN electron microscope (FEI, Eindoven, The Netherlands) operating at $100 \mathrm{kV}$. Digital acquisitions were made with a bottom-mounted Keen View 1K CCD camera (Soft Imaging System, Muenster, Germany).

\section{Attenuated Total Reflectance Fourier-Transform Infrared Spectroscopy and Post-Run Spectra Computations}

A $10-\mu \mathrm{l}$ drop of the NT-proBNP heptapeptide-analogue, KMVLYTL, fibril suspension was cast on a front-coated Au mirror and left to dry slowly at ambient conditions to form a thin film. Infrared spectra were obtained from these films at a resolution of 4 $\mathrm{cm}^{-1}$, utilizing an IR microscope (IRScope II by Bruker Optics) equipped with a Ge attenuated total reflectance (ATR) objective lens $(20 \times)$ and attached to a Fourier-transform infrared (FTIR) spectrometer (Equinox 55 by Bruker Optics).

Internal reflection spectroscopy has several advantages compared with the more common $\mathrm{KBr}$ dispersion technique. ${ }^{51}$ The choice of ATR was dictated by the need to exclude any possible spectroscopic and chemical interactions between the sample and the dispersing medium. Having a penetration depth ca. $1-2 \mu \mathrm{m}\left(1000 \mathrm{~cm}^{-1}, \mathrm{Ge}\right)$, ATR is free of saturation effects, which may be present in the transmission spectra of thicker samples. Moreover, the use of a single reflection accessory facilitates the acquisition of data from small samples. Ten 32scan spectra were collected from each sample and averaged to improve the $\mathrm{S} / \mathrm{N}$ ratio. The spectra are shown in the absorption mode after correction for the wavelength dependence of the penetration depth ( $\mathrm{pd} \propto$ 2). Absorption peak maxima were determined from the minima in the second derivative of the corresponding spectra. Derivatives were computed analytically using routines of the Bruker OPUS/OS2 software and included smoothing by the Savitzky-Golay algorithm over a +/$8 \mathrm{~cm}^{-1}$ range, around each data point. ${ }^{52}$ Smoothing over narrower ranges resulted in a deterioration of the $\mathrm{S} / \mathrm{N}$ ratio and did not increase the number of minima that could be determined with confidence.

\section{Congo Red Staining and Polarized Light Microscopy}

The NT-proBNP heptapeptide-analogue, KMVLYTL, fibril suspensions were applied to glass slides and stained with a $10 \mathrm{mM}$ Congo Red (Sigma) solution in phosphate-buffered saline ( $\mathrm{pH} \mathrm{7.4)} \mathrm{for} \sim 2 \mathrm{~h}$ and left to dry. Then, they were washed several times with $90 \%$ ethanol and left to dry. The samples were observed, respectively, both under bright 


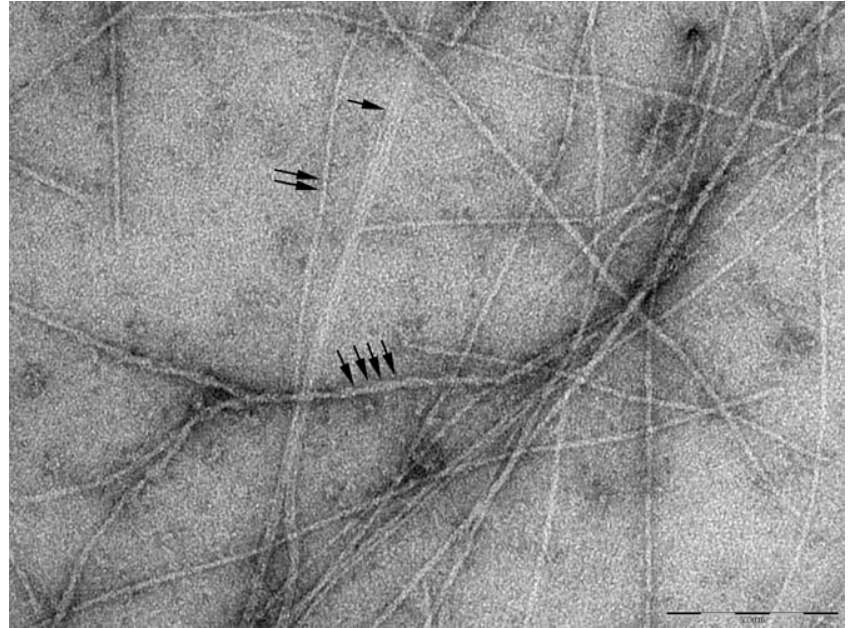

FIGURE 2 Electron micrographs of amyloid-like fibrils derived by self-assembly, from a $15 \mathrm{mg} \mathrm{ml}^{-1}$ solution of the NT-proBNP peptide-analogue KMVLYTL in distilled water, $\mathrm{pH}$ 5.5. Fibrils were negatively stained with $1 \%$ uranyl acetate. The fibrils exhibit a characteristic for amyloid-like fibrils polymorphism. ${ }^{53-56}$ The thinner protofilaments, of indeterminate length (several microns), unbranched, $\sim 50-60 \AA$ in diameter, have a tendency to coalesce laterally and in register among each other into nontwisted ribbons (single arrow). A varying number of such protofilaments are frequently wound around each other, forming various supercoils, with different diameters (double and multiple arrows). These aggregates of protofilaments and fibrils with a higher order structure, ultimately, form gels (data not shown). Bar $200 \mathrm{~nm}$.

field illumination and between crossed polars using a Leica MZ75 polarizing stereomicroscope equipped with a JVC GC-X3E camera.

\section{RESULTS}

The synthesized NT-proBNP peptide-analogue, KMVLYTL, self-assembles into amyloid-like fibrils (see Figure 2) after 12 week incubation in distilled water (see the "Materials and Methods" section) and forms fibril-containing gels. The fibrils were judged to be amyloid-like from their tinctorial and structural characteristics. Electron micrographs show that they are linear unbranched fibrils of indeterminate length and uniform in diameter (ca. 50-60 ^) (Figure 2, single arrow). Occasionally, they tend to aggregate laterally and in register (Figure 2, single arrow), forming ribbons of parallel/antiparallel fibrils, which may extend under, presumably, favorable conditions, to form planar extended surfaces of parallel/antiparallel and in register fibrils. More frequently, they tend to wind around each other, forming supercoils (multistranded cables) of different diameters (Figure 2, multiple arrows). The simplest such supercoils (multistranded cables) are double helices (Figure 2, double arrows). This morphological polymorphism was observed with many different amyloid-forming peptides and proteins using, usu- ally, electron microscopy and may be related to amyloid fibril assembly pathways and cytotoxicity mechanisms. ${ }^{53-56}$ Oriented fibers formed from suspensions of these fibrils give oriented X-ray diffraction patterns (see Figure 3), which closely resemble typical "cross- $\beta$ " patterns from amyloid fibrils ${ }^{57-59}$ : a strong meridional reflection at $4.75 \AA$, representing the repetitive interchain distance between hydrogen-bonded $\beta$ strands and a strong equatorial reflection at $10.40 \AA$, corresponding to the intersheet stacking/packing periodicity. The strong meridional reflection at $4.75 \AA$ suggests that the $\beta$ sheets are oriented with their $\beta$-strands perpendicular to the fiber axis (and also to the long axis of the amyloid-like fibrils), whereas the strong equatorial reflection at $10.40 \AA$ corresponds to the intersheet distance, suggesting that the packing/stacking of the $\beta$-sheets is parallel to the fiber axis and preferentially oriented. Concomitant evidence of the existence of an antiparallel $\beta$-sheet structure in the amyloidlike fibrils formed from the synthesized NT-proBNP peptideanalogue KMVLYTL comes from ATR FTIR spectroscopy. An ATR FTIR spectrum of a thin film cast from suspensions of the amyloid-like fibrils of the synthesized NT-proBNP

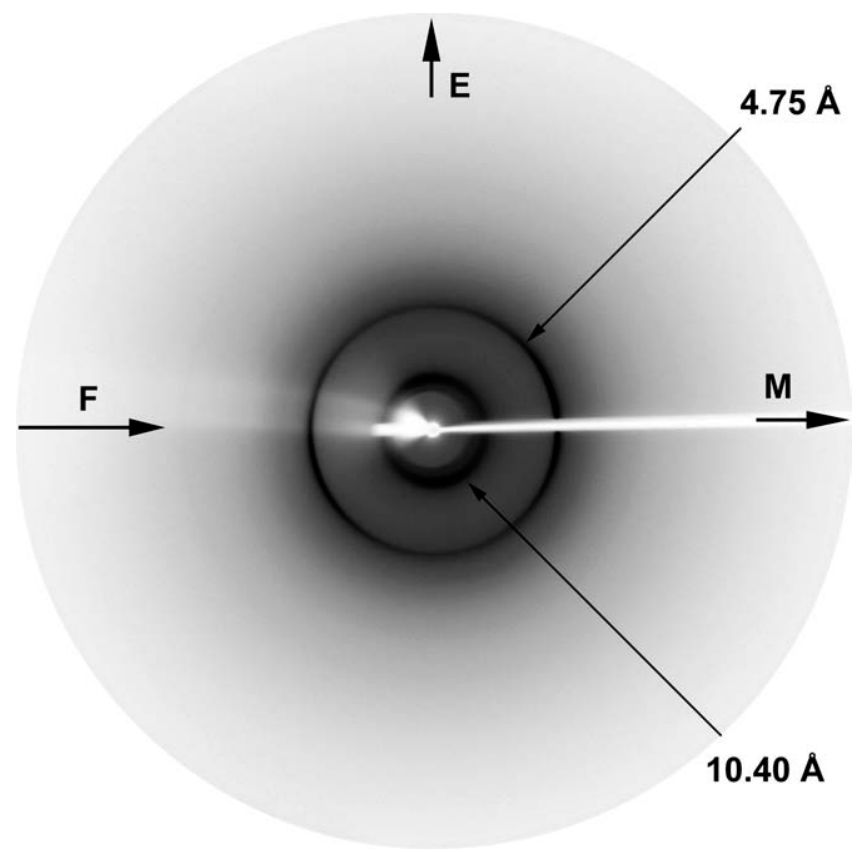

FIGURE 3 X-ray diffraction pattern from an oriented fiber of NTproBNP heptapeptide-analogue, KMVLYTL, derived amyloid-like fibrils. The meridian (direction parallel to the fiber axis) is horizontal, and the equator is vertical in this display. The X-ray diffraction pattern resembles closely a "cross- $\beta$ " pattern showing a strong meridional $4.75 \AA$ reflection and a strong $10.40 \AA$ reflection on the equator. The structural repeat of $4.75 \AA$ corresponds to the spacing of adjacent, hydrogen bonded, $\beta$-strands, perpendicular to the fiber axis, and the $10.40 \AA$ spacing corresponds to the face-to-face separation (packing and stacking distance) of the $\beta$-sheets, parallel to the fiber axis. 


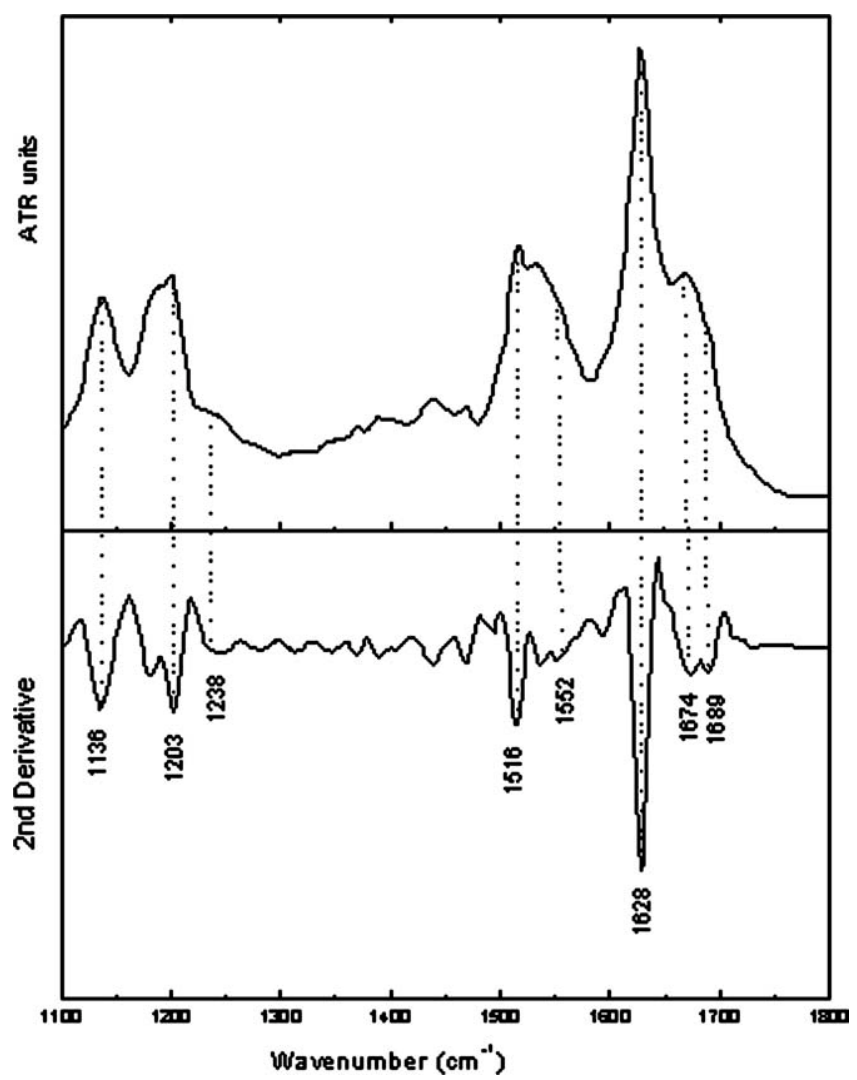

FIGURE 4 ATR FTIR $\left(900-1800 \mathrm{~cm}^{-1}\right)$ spectrum of NT-proBNP heptapeptide-analogue, KMVLYTL, derived amyloid fibrils cast as a thin film on a Au mirror (see the "Materials and Methods" section). Error bar equals $0.5 \sigma$ in the IR spectrum.

peptide-analogue KMVLYTL is shown in Figure 4. The spectrum (Figure 4, Table I) shows prominent bands at $1628 \mathrm{~cm}^{-1}, 1552 \mathrm{~cm}^{-1}$, and $1238 \mathrm{~cm}^{-1}$ in the amide I, II, and III regions, respectively. These bands, undoubtedly, are due to $\beta$-sheet conformation. ${ }^{60-63}$ The high wavenumber band at $1689 \mathrm{~cm}^{-1}$, seen as a shoulder in the amide I band of the ATR spectrum (Figure 4, Table I), is a strong indication that the $\beta$-sheets are antiparallel. ${ }^{60-63}$ The fibrils formed by the synthesized NT-proBNP peptide-analogue KMVLYTL bind the dye Congo Red showing the characteristic for amyloid fibrils apple/green birefringence in a polarizing microscope, under crossed polars (see Figure 5). ${ }^{64}$ Thus, they also satisfy the third criterion characterizing amyloid-like fibrils.

\section{DISCUSSION}

Amyloids can be formed by a diverse group of proteins or peptides. There is no apparent similarity in the biological role, 3D structure, amino acid sequence, molecular weight, or morphology between amyloidogenic proteins. Additionally, several proteins and peptides, which are not associated with amyloidoses, have been proven to form amyloid-like fibrils in vitro, indicat- ing that amyloid formation may be an inherent ability of proteins in some surrounding conditions/environments. ${ }^{1,2}$ However, recent evidence supports a sequence propensity in the formation of amyloid fibrils. ${ }^{44-46}$ Such short-sequence stretches of a protein that are responsible for initiating amyloid aggregation are, frequently, referred to as "aggregation-prone" amyloid forming regions or "amyloidogenic determinants." 47 The presence of these amyloidogenic determinants is sometimes linked with mutations resulting to a number of amyloidoses, or it is related to molecular evolution in the case of functional amyloids. Amyloidogenic determinants, usually short peptides, often act as "conformational switches" and thus act as templates initiating amyloid formation through, perhaps, structural rearrangements. ${ }^{47}$ The identification of amyloidogenic determinants in proteins that are connected with some kind of amyloidosis may help in our understanding of the molecular mechanisms of the disease, as well as they may indicate therapeutic approaches for the treatment of amyloidoses. Recent, elegant, and exciting works by Kelly and coworkers announced encouraging results from phase II/III clinical trials of compounds that prevent protein misfolding and aggregation in people with transthyretin amyloidoses. ${ }^{65-67}$ In this respect, it is perhaps interesting to mention our recent finding that nearly all experimentally verified amyloidogenic determinants in proteins responsible for amyloidoses reside on the surface of the solved relevant protein structures, directly accessible to stabilization by suitably designed compounds/drugs. ${ }^{47}$

In the case of cardiac amyloidoses, and especially in IAA, there has been proof of the possibility either BNP, ANP, or its precursor peptide (proANP) to be constituents of the amyloid fibrils. ${ }^{8}$ The greater elevation of the secretion of proBNP compared with proANP, in regions of the heart adjacent to amyloid fibrils, leads to a increased concentration in blood of BNP and NT-proBNP. ${ }^{10}$ Also, recent studies show that it is very common when a hormone participates in the formation

Table I Bands Observed in an ATR FTIR Spectrum Taken From a Thin Film, Formed From a NT-proBNP HeptapeptideAnalogue, KMVLYTL, Amyloid-Like Fibril Suspension, Cast on a Front-Coated Au Mirror, and Their Assignments

\begin{tabular}{ll}
\hline Band $\left(\mathbf{c m}^{-\mathbf{1}}\right)$ & \multicolumn{1}{c}{ Assignment $^{\mathbf{6 0 - 6 3 , 7 6}}$} \\
\hline 1136 & TFA \\
1203 & TFA \\
1238 & Amide III ( $\beta$-sheet) \\
1469 & $\mathrm{CH}_{2}$ scissoring \\
1516 & Tyr \\
1552 & Amide II $(\beta$-sheet) \\
1628 & Amide I ( $\beta$-sheet) \\
1674 & TFA \\
1689 & Amide I (antiparallel $\beta$-sheet) \\
\hline
\end{tabular}




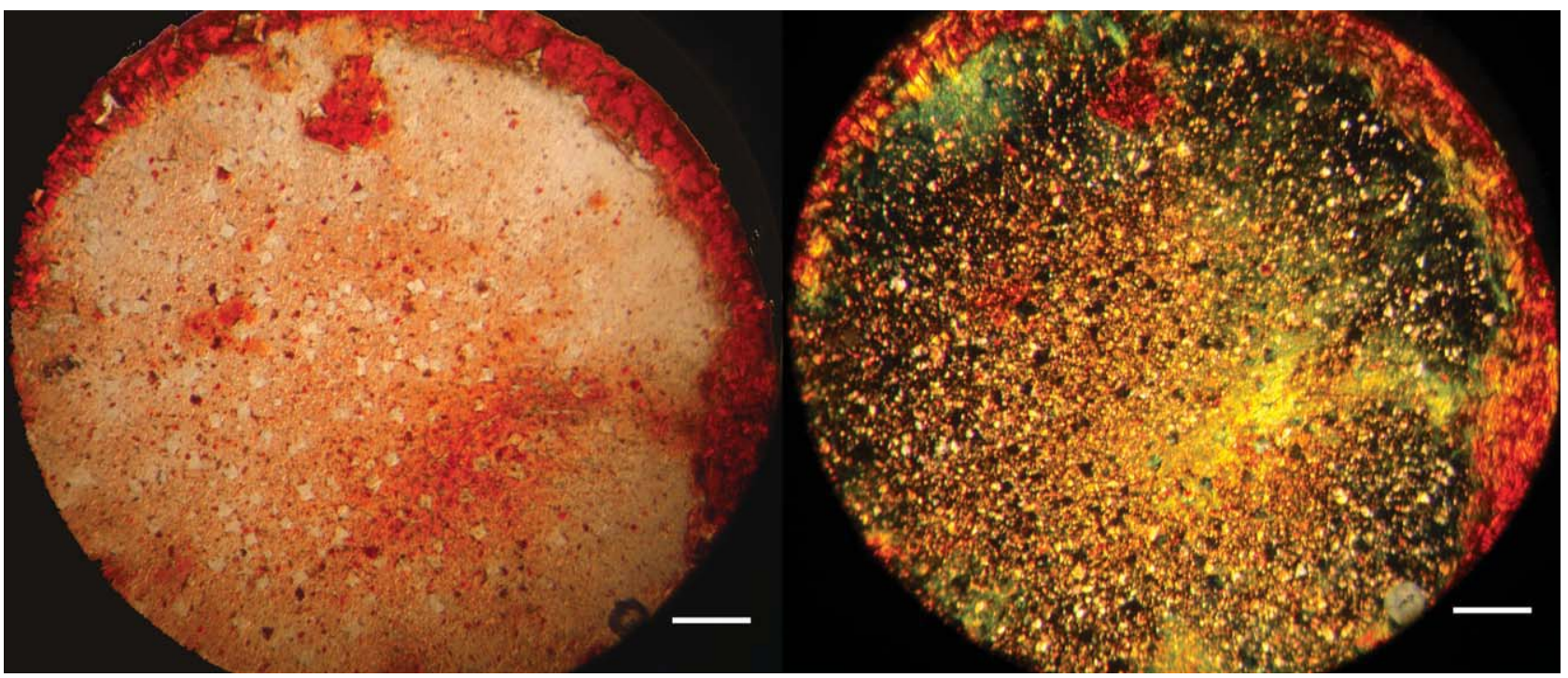

FIGURE 5 Photomicrographs of NT-proBNP heptapeptide-analogue, KMVLYTL, derived fibrils stained with Congo red: (a) bright field illumination and (b) crossed polars. The redapple/green birefringence characteristic for amyloid fibrils is seen. Bar $100 \mu \mathrm{m}$.

of amyloid fibrils, these fibrils are to be localized in the organ where the hormone is secreted. ${ }^{60}$ So the increase of the concentration of BNP and NT-proBNP as well as the expectance of amyloid formation in the heart give rise to a number of questions involving whether proBNP (or NT-proBNP) has an important role in the formation of amyloids in the heart.

The heptapeptide (KMVLYTL) synthesized in this work self assembles into amyloid-like fibrils that exhibit the three characteristic features of amyloids. The fact that this peptide forms amyloid-like fibrils in vitro indicates that it is an amyloidogenic determinant of proBNP and especially of the NTproBNP sequence, even after the maturation of the prohormone (proBNP). This shows the predisposition/inherent ability of NT-proBNP and proBNP to form amyloid fibrils in certain environmental conditions. This finding leads to a possible participation of these molecules in the formation and self-assembly of amyloid fibrils in the heart in IAA, as has been found for proANP ${ }^{8}$ and, also, in atrial fibrillation, ${ }^{68,69}$ thus perhaps suggesting a possible biological role for these peptides for the first time. Furthermore, it does not exclude the involvement of proBNP and NT-proBNP in other types of cardiac amyloidoses, such as the amyloidosis due to AL light chains ${ }^{43,70}$ and also familial and senile amyloidoses due to mutant transthyretin and wild-type transthyretin, respectively. ${ }^{70} \mathrm{It}$ is worth mentioning that NT-proBNP is a sensitive marker of myocardial dysfunction in $\mathrm{AL}$ amyloidosis. ${ }^{43}$ However, it should be clearly stated here that there is no prior evidence for this involvement, neither theoretical nor experimental. We simply make this speculative assumption to attract experimental efforts, which should aim at clarifying this possible involve- ment. It should, perhaps, be mentioned at this point that the corresponding sequence in proANP and NT-proANP, KLRALLT, predicted also by AMYLPRED ${ }^{47}$ to be an amyloidogenic determinant in the ANP relevant sequences, also exhibits amyloidogenic determinant characteristics, indicating the possible involvement of this natriuretic peptide prohormone too in cardiac amyloidoses (Iconomidou and Hamodrakas, In preparation). The question, which of course arises, is how the mature BNP and ANP are also involved in cardiac amyloidoses. This is a matter of an ongoing investigation (Iconomidou and Hamodrakas, unpublished).

At this point, it is perhaps rewarding to discuss prominent molecular features of NT-proBNP, which may be important
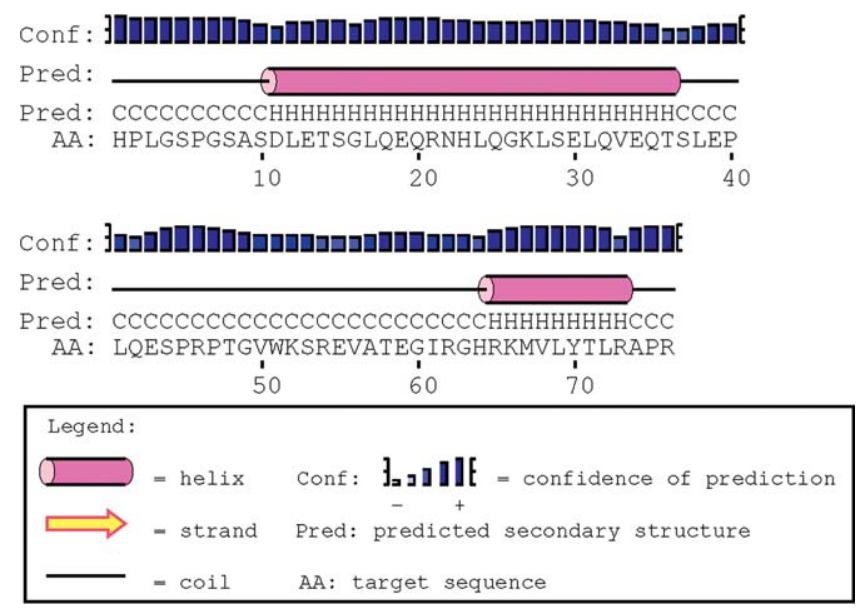

FIGURE 6 Secondary structure prediction of NT-proBNP (AA: target sequence), utilizing the algorithm PSIPRED ${ }^{71}$ (Pred: predicted secondary structure, $\mathrm{H}=\alpha$-helix, $\mathrm{E}=\beta$-strand, $\mathrm{C}=$ random coil). 
for its ability to self-assemble and form amyloid-like fibrils. Secondary structure prediction of NT-proBNP, utilizing the algorithm PSIPRED, ${ }^{71}$ indicates that the secondary structure of NT-proBNP is dominated by the presence of two $\alpha$-helices (see Figure 6): a long $\alpha$-helix formed by residues $10-36$ (see Figure 6), which correspond to residues 36-62 of Figure 1 and a second, shorter $\alpha$-helix formed by residues 64-73 (see Figure 6), which correspond to residues 90-99 of Figure 1, and which encompasses the amyloidogenic determinant KMVLYTL predicted by AMYLPRED. ${ }^{47}$ Similar secondary structure prediction results were obtained applying two other popular secondary structure prediction algorithms, JPRED ${ }^{72}$ and PORTER ${ }^{73}$ (data not shown). However, the use of our consensus secondary structure prediction algorithm SECSTR, ${ }^{74}$ although it suggests a possible, rather long $\alpha$-helix, between residues $10-36$ of Figure 6 (which correspond to residues 36-62 of Figure 1) and also predicts the second shorter $\alpha$-helix formed by residues 64-73 (see Figure 6), which correspond to residues 90-99 of Figure 1, in agreement with the above mentioned popular and accurate prediction algorithms, it also suggests an alternative, stronger prediction for a $\beta$-strand (five methods out of six predict a $\beta$-strand as opposed to three methods out of six that predict an $\alpha$-helix-the algorithm is capable of predicting simultaneously more than one possible type of secondary structure for the same sequence) for the amyloidogenic determinant KMVLYTL (residues 66-72 of Figure 6/residues 92-98 of Figure 1). Thus, it implies that this amyloidogenic determinant sequence KMVLYTL has all the distinctive features of a "chameleon" sequence ${ }^{47}$ (a sequence with the ability to act as "conformational switch" forming either an $\alpha$-helix or a $\beta$ strand depending on the environmental conditions) and may decisively drive and dictate the amyloid fibrillation process. In an attempt to find out whether the "isolated" KMVLYTL peptide undergoes, indeed, a conformational transition from $\alpha$ helix to $\beta$-sheet during the amyloid fibril formation time-period, no such transition was observed: the "isolated" KMVLYTL peptide adopts an antiparallel $\beta$-sheet conformation immediately after dissolving it in water (data not shown).

In a piece of elegant theoretical and experimental work over a decade ago, Richards and coworkers ${ }^{75}$ suggested that the portion of the NT-proBNP or proBNP molecule, which corresponds to the long $\alpha$-helix (residues 36-62 of Figure 1, 10-36 of Figure 6), marked by the presence of the underlined leucine $(\mathrm{L})$ residues in Figure 1, is responsible for proBNP and proANP oligomerization through leucine zipper-like coiledcoil (supercoiled helices of $\alpha$-helices) motifs. Therefore, the picture emerging from their inspired theoretical and experimental work ${ }^{75}$ and from the experimental work in this study is that NT-proBNP and proBNP (respectively NT-proANP and proANP) oligomerize via leucine zipper-like coiled-coil motifs and further polymerize into amyloid-like fibrils through KMVLYTL $\beta$-strand interactions into $\beta$-sheets.

Consequently, if attempts will be made in the future toward designing remedies for IAA or other cardiac amyloidoses, which involve the precursor molecules of natriuretic peptides of BNP and ANP, following the impressive paradigm of Kelly and coworkers, ${ }^{65-67}$ efforts should be focused (a) in designing molecules that prevent KMVLYTL polymerization and (b) in designing molecules that prevent oligomerization through the leucine zipper-like coiled-coil motifs.

\section{CONCLUSIONS}

In this study, utilizing our algorithm AMYLPRED, ${ }^{47}$ we predicted from the primary structure of NT-proBNP (or proBNP), a unique, specific, amyloidogenic determinant (amyloid-forming region) sequence, KMVLYTL, located near the C-terminal of NT-proBNP. We have synthesized this peptide and we have shown, utilizing several experimental methods, that it forms amyloid-like fibrils in vitro. The consequences of this finding in IAA, atrial fibrillation, and other cardiac amyloidoses are discussed, and hypothetical possible targets for small molecules/drugs designed to prevent aggregation and amyloid fibril formation are indicated.

The authors thank Peter Everitt for his excellent technical assistance and their collaborators Dr. G. Chryssikos and Dr. V. Gionis for help with the experiments. They also thank the reviewers of this manuscript for their useful and constructive criticism.

\section{REFERENCES}

1. Chiti, F.; Dobson, C. M. Annu Rev Biochem 2006, 75, 333-366.

2. Uversky, V. N.; Fink, A. L. Biochim Biophys Acta 2004, 1698, 131-153.

3. Kelly, J. W.; Balch, W. E. J Cell Biol 2003, 161, 461-462.

4. Stevens, F. J. Amyloid 2004, 11, 232-244.

5. Iconomidou, V. A.; Vriend, G.; Hamodrakas, S. J. FEBS Lett 2000, 479, 141-145.

6. Iconomidou, V. A.; Hamodrakas, S. J. Curr Protein Pept Sci 2008, 9, 291-309.

7. Johansson, B.; Wernstedt, C.; Westermark, P. Biochem Biophys Res Commun 1987, 148, 1087-1092.

8. Pucci, A.; Wharton, J.; Arbustini, E.; Grasso, M.; Diegoli, M.; Needleman, P.; Vigano, M.; Polak, J. M. J Pathol 1991, 165, 235-241.

9. Maredia, N.; Ray, S. G. Clin Med 2005, 5, 504-509.

10. Takemura, G.; Takatsu, Y.; Doyama, K.; Itoh, H.; Saito, Y.; Koshiji, M.; Ando, F.; Fujiwara, T.; Nakao, K.; Fujiwara, H. J Am Coll Cardiol 1998, 31, 754-765.

11. Kaye, G. C.; Butler, M. G.; d'Ardenne, A. J.; Edmondson, S. J.; Camm, A. J.; Slavin, G. Br Heart J 1986, 56, 317-320.

12. Murthy, K. K.; Thibault, G.; Garcia, R.; Gutkowska, J.; Genest, J.; Cantin, M. Biochem J 1986, 240, 461-469. 
13. de Bold, A. J.; Borenstein, H. B.; Veress, A. T.; Sonnenberg, H. Life Sci 1981, 28, 89-94.

14. de Bold, A. J. Science 1985, 230, 767-770.

15. Kangawa, K.; Matsuo, H. Biochem Biophys Res Commun 1984, 118, 131-139.

16. Sudoh, T.; Kangawa, K.; Minamino, N.; Matsuo, H. Nature 1988, 332, 78-81.

17. Yasue, H.; Yoshimura, M.; Sumida, H.; Kikuta, K.; Kugiyama, K.; Jougasaki, M.; Ogawa, H.; Okumura, K.; Mukoyama, M.; Nakao, K. Circulation 1994, 90, 195-203.

18. Mair, J. Clin Chem Lab Med 2008, 46, 1507-1514.

19. Brenner, B. M.; Ballermann, B. J.; Gunning, M. E.; Zeidel, M. L. Physiol Rev 1990, 70, 665-699.

20. Kambayashi, Y.; Nakao, K.; Mukoyama, M.; Saito, Y.; Ogawa, Y.; Shiono, S.; Inouye, K.; Yoshida, N.; Imura, H. FEBS Lett 1990, 259, 341-345.

21. Sudoh, T.; Maekawa, K.; Kojima, M.; Minamino, N.; Kangawa, K.; Matsuo, H. Biochem Biophys Res Commun 1989, 159, 1427-1434.

22. Sawada, Y.; Suda, M.; Yokoyama, H.; Kanda, T.; Sakamaki, T.; Tanaka, S.; Nagai, R.; Abe, S.; Takeuchi, T. J Biol Chem 1997, 272, 20545-20554.

23. Yan, W.; Wu, F.; Morser, J.; Wu, Q. Proc Natl Acad Sci U S A 2000, 97, 8525-8529.

24. McDonagh, T. A.; Robb, S. D.; Murdoch, D. R.; Morton, J. J.; Ford, I.; Morrison, C. E.; Tunstall-Pedoe, H.; McMurray, J. J.; Dargie, H. J. Lancet 1998, 351, 9-13.

25. Mukoyama, M.; Nakao, K.; Saito, Y.; Ogawa, Y.; Hosoda, K.; Suga, S.; Shirakami, G.; Jougasaki, M.; Imura, H. Lancet 1990, 335, 801-802.

26. Omland, T.; Aakvaag, A.; Bonarjee, V. V.; Caidahl, K.; Lie, R. T.; Nilsen, D. W.; Sundsfjord, J. A.; Dickstein, K. Circulation 1996, 93, 1963-1969.

27. Tsutamoto, T.; Wada, A.; Maeda, K.; Hisanaga, T.; Maeda, Y.; Fukai, D.; Ohnishi, M.; Sugimoto, Y.; Kinoshita, M. Circulation 1997, 96, 509-516.

28. Kremastinos, D. T.; Hamodraka, E.; Parissis, J.; Tsiapras, D.; Dima, K.; Maisel, A. Am Heart J 2010, 159, 68-74.

29. Hunt, P. J.; Yandle, T. G.; Nicholls, M. G.; Richards, A. M.; Espiner, E. A. Biochem Biophys Res Commun 1995, 214, 1175-1183.

30. Shimizu, H.; Masuta, K.; Asada, H.; Sugita, K.; Sairenji, T. Clin Chim Acta 2003, 334, 233-239.

31. Valli, N.; Gobinet, A.; Bordenave, L. J Lab Clin Med 1999, 134, 437-444.

32. Vanderheyden, M.; Bartunek, J.; Goethals, M. Eur J Heart Fail 2004, 6, 261-268.

33. McCullough, P. A.; Omland, T.; Maisel, A. S. Rev Cardiovasc Med 2003, 4, 72-80.

34. Wu, A. H.; Smith, A. Eur J Heart Fail 2004, 6, 355-358.

35. Hall, C. Eur J Heart Fail 2004, 6, 257-260.

36. Hall, C. J Card Fail 2005, 11, S81-S83.

37. Richards, A. M. Hypertension 2007, 50, 25-30.

38. Martinez-Rumayor, A.; Richards, A. M.; Burnett, J. C.; Januzzi, J. L., Jr. Am J Cardiol 2008, 101, 3-8.

39. Pandey, K. N. J Am Soc Hypertens 2008, 2, 210-226.

40. Palmer, S. C.; Yandle, T. G.; Nicholls, M. G.; Frampton, C. M.; Richards, A. M. Eur J Heart Fail 2009, 11, 832-839.

41. Palmer, S. C.; Richards, A. M. J Am Coll Cardiol 2009, 53, 891-892.

42. Sisson, D. J Vet Cardiol 2009, 11 Suppl 1, S5-S7.
43. Palladini, G.; Campana, C.; Klersy, C.; Balduini, A.; Vadacca, G.; Perfetti, V.; Perlini, S.; Obici, L.; Ascari, E.; d'Eril, G. M.; Moratti, R.; Merlini, G. Circulation 2003, 107, 2440-2445.

44. Esteras-Chopo, A.; Serrano, L.; Lopez de la Paz, M. Proc Natl Acad Sci U S A 2005, 102, 16672-16677.

45. Lopez de la Paz, M.; Serrano, L. Proc Natl Acad Sci U S A 2004 , 101, 87-92.

46. Teng, P. K.; Eisenberg, D. Protein Eng Des Sel 2009, 22, 531-536.

47. Frousios, K. K.; Iconomidou, V. A.; Karletidi, C. M.; Hamodrakas, S. J. BMC Struct Biol 2009, 9, 44.

48. Westermark, P. Amyloid 1994, 1, 47-60.

49. He, X. L.; Dukkipati, A.; Garcia, K. C. J Mol Biol 2006, 361, 698-714.

50. The CCP4 Suite: Programs for Protein Crystallography by Collaborative Computational Project, Number 4, Acta Cryst 1994, D50, 760-763.

51. de Jongh, H. H.; Goormaghtigh, E.; Ruysschaert, J. M. Anal Biochem 1996, 242, 95-103.

52. Savitsky, A.; Golay, M. Anal Chem 1964, 36, 13.

53. Kodali, R.; Wetzel, R. Curr Opin Struct Biol 2007, 17, 48-57.

54. Kreplak, L.; Aebi, U. Adv Protein Chem 2006, 73, 217-233.

55. Pedersen, J. S.; Andersen, C. B.; Otzen, D. E. FEBS J 2010, 277, 4591-4601.

56. Goldsbury, C.; Baxa, U.; Simon, M. N.; Steven, A. C.; Engel, A.; Wall, J. S.; Aebi, U.; Muller, S. A. J Struct Biol 2011, 173, 1-13.

57. Geddes, A. J.; Parker, K. D.; Atkins, E. D.; Beighton, E. J Mol Biol 1968, 32, 343-358.

58. Fraser, R.; MacRae, T. Conformation in Fibrous Proteins and Related Synthetic Polypeptides; Academic Press: New York, 1973.

59. Jahn, T. R.; Makin, O. S.; Morris, K. L.; Marshall, K. E.; Tian, P.; Sikorski, P.; Serpell, L. C. J Mol Biol 2010, 395, 717-727.

60. Haris, P. I.; Chapman, D. Biopolymers 1995, 37, 251-263.

61. Jackson, M.; Mantsch, H. H. Crit Rev Biochem Mol Biol 1995, $30,95-120$

62. Krimm, S.; Bandekar, J. Adv Protein Chem 1986, 38, 181-364.

63. Surewicz, W. K.; Mantsch, H. H.; Chapman, D. Biochemistry 1993, 32, 389-394.

64. Sunde, M.; Blake, C. Adv Protein Chem 1997, 50, 123-159.

65. Choi, S.; Reixach, N.; Connelly, S.; Johnson, S. M.; Wilson, I. A.; Kelly, J. W. J Am Chem Soc 2010, 132, 1359-1370.

66. Connelly, S.; Choi, S.; Johnson, S. M.; Kelly, J. W.; Wilson, I. A. Curr Opin Struct Biol 2010, 20, 54-62.

67. Miller, G. Science 2009, 326, 1337-1339.

68. Goette, A.; Rocken, C. Eur Heart J 2004, 25, 1185-1186.

69. Rocken, C.; Peters, B.; Juenemann, G.; Saeger, W.; Klein, H. U.; Huth, C.; Roessner, A.; Goette, A. Circulation 2002, 106, 2091-2097.

70. Falk, R. H.; Dubrey, S. W. Prog Cardiovasc Dis 2010, 52, 347-361.

71. Bryson, K.; McGuffin, L. J.; Marsden, R. L.; Ward, J. J.; Sodhi, J. S.; Jones, D. T. Nucleic Acids Res 2005, 33, W36-W38.

72. Cole, C.; Barber, J. D.; Barton, G. J. Nucleic Acids Res 2008, 36, W197-W201.

73. Bau, D.; Martin, A. J.; Mooney, C.; Vullo, A.; Walsh, I.; Pollastri, G. BMC Bioinformatics 2006, 7, 402.

74. Hamodrakas, S. J. Comput Appl Biosci 1988, 4, 473-477.

75. Seidler, T.; Pemberton, C.; Yandle, T.; Espiner, E.; Nicholls, G.; Richards, M. Biochem Biophys Res Commun 1999, 255, 495-501.

76. Valenti, L. E.; Paci, M. B.; De Pauli, C. P.; Giacomelli, C. E. Anal Biochem 2011, 410, 118-123. 